\title{
Exponential Stability of Impulsive Delay Differential Equations
}

\author{
G. L. Zhang, M. H. Song, and M. Z. Liu \\ Department of Mathematics, Harbin Institute of Technology, Harbin 150001, China \\ Correspondence should be addressed to M. H. Song; songmh@lsec.cc.ac.cn
}

Received 8 September 2012; Revised 3 January 2013; Accepted 8 January 2013

Academic Editor: Xinan Hao

Copyright (C) 2013 G. L. Zhang et al. This is an open access article distributed under the Creative Commons Attribution License, which permits unrestricted use, distribution, and reproduction in any medium, provided the original work is properly cited.

\begin{abstract}
The main objective of this paper is to further investigate the exponential stability of a class of impulsive delay differential equations. Several new criteria for the exponential stability are analytically established based on Razumikhin techniques. Some sufficient conditions, under which a class of linear impulsive delay differential equations are exponentially stable, are also given. An Euler method is applied to this kind of equations and it is shown that the exponential stability is preserved by the numerical process.
\end{abstract}

\section{Introduction}

Impulsive differential equations arise widely in the study of medicine, biology, economics, engineering, and so forth. In recent years, theory of impulsive differential delay equations (IDDEs) has been an object of active research (see [1-18] and references therein). The results about the existence and uniqueness of IDDEs have been studied in $[2,7]$. The stability of IDDEs has attracted increasing interest in both theoretical research and practical applications (see [1, 3, 5-18] and references therein). In particular, special attention has been focused on exponential stability of IDDEs (see [1, 3, 8, 9, 15]) because it has played an important role in many areas.

There is a little work done on exponential stability for IDDEs by the Lyapunov-Razumikhin method. Wang and Liu [15] have extended Lyapunov-Razumikhin method to IDDEs and established some exponential stability criteria. In this paper we restrict the length of each impulsive interval instead of some conditions in [15]. As a result, several new criteria on exponential stability are analytically derived.

There are a few papers on numerical methods of impulsive differential equations. In [19], Covachev et al. obtained a convergent difference approximation for a nonlinear impulsive ordinary system in a Banach space. In [20, 21], the authors studied the stability of Runge-Kutta methods for linear impulsive ordinary differential equations. In [4], Ding et al. studied the convergence property of an Euler method for IDDEs. In [18], asymptotic stability of numerical solutions and exact solutions of a class of linear IDDEs was studied by the property of DDEs without impulsive perturbations. The convergence of the numerical methods for this kind of equations was studied. In this paper, we study exponential stability of the numerical solutions of linear IDDEs.

The rest of the paper is organized as follows. In Section 2, we obtained two criteria on exponential stability for IDDEs by the Lyapunov-Razumikhin method. The results obtained are applied to a class of linear IDDEs. In the last section, we prove that the Euler method for the linear IDDEs preserves the analytic exponential stability.

\section{Stability of Analytic Solutions}

Consider the impulsive delay differential system

$$
\begin{aligned}
& x^{\prime}(t)=f\left(t, x_{t}\right), \quad t \in\left[t_{k-1}, t_{k}\right), k=1,2,3, \ldots, \\
& \Delta x(t)=I_{k}\left(t, x_{t^{-}}\right), \quad t=t_{k}, k=1,2,3, \ldots,
\end{aligned}
$$

where $f: R_{+} \times \mathrm{PC}\left([-\tau, 0], R^{d}\right) \rightarrow R^{d} ; I_{k}: \mathrm{PC}\left([-\tau, 0], R^{d}\right) \rightarrow$ $R^{d} ; 0 \leq t_{0}<t_{1}<t_{2}<\cdots<t_{k}<\cdots$, with $t_{k} \rightarrow \infty$ as $k \rightarrow$ $\infty ; \mathrm{PC}\left([-\tau, 0], R^{d}\right)$ is a set of piecewise continuous functions $g(t)$ which have a finite number of points of discontinuity in a finite interval and $g(t)=g\left(t^{+}\right)$for all $t$. We assume that $f(t, 0) \equiv 0, I_{k}(t, 0) \equiv 0$, so that $x \equiv 0$ is a solution of $(1)$ as $x_{t_{0}}=\Phi \equiv 0$, which we call the zero solution. 
Definition 1 (see [15]). A function $V: R_{+} \times R^{d} \rightarrow R_{+}$is said to belong to the class $v_{0}$ if

(i) $V$ is continuous in each of the sets $\left[t_{k-1}, t_{k}\right) \times R^{d}$ and for each $x \in R^{d}, t \in\left[t_{k-1}, t_{k}\right), \lim _{(t, y) \rightarrow\left(t_{k}^{-}, x\right)} V(t, y)=$ $V\left(t_{k}^{-}, x\right)$ exists, $k=1,2, \ldots$;

(ii) $V(t, x)$ is locally Lipschitzian in all $x \in R^{d}$, and for all $t \geq t_{0}, V(t, 0) \equiv 0$.

Definition 2 (see [15]). Given a function $V: R_{+} \times R^{d} \rightarrow R_{+}$, the upper right-hand derivative of $V$ with respect to system (1) is defined by

$D^{+} V(t, \Psi(0))$

$$
=\limsup _{h \rightarrow 0^{+}} \frac{1}{h}[V(t+h, \Psi(0)+h f(t, \Psi))-V(t, \Psi(0))],
$$

for $(t, \Psi) \in R_{+} \times \operatorname{PC}\left([-\tau, 0], R^{d}\right)$.

Definition 3 (see [15]). The zero solution of (1) is said to be exponentially stable, if there exist constants $\lambda>0$ and $M \geq 1$, such that for any initial data $x_{t_{0}}=\Phi \in \operatorname{PC}\left([-\tau, 0], R^{d}\right)$,

$$
\left\|x\left(t, t_{0}, \Phi\right)\right\| \leq M\|\Phi\|_{\tau} e^{-\lambda\left(t-t_{0}\right)}, \quad t \geq t_{0} .
$$

Theorem 4. Assume that there exist a function $V \in v_{0}$; constants $d_{k}>-1, k=1,2, \ldots$; positive constants $C_{1}, C_{2}, \lambda, l_{1}$; and a function $\left.m(t) \in P Q\left(t_{0}-\tau, \infty\right), R_{+}\right)$with inf $\operatorname{int}_{t \geq \tau} m(t) \geq$ $\lambda$, such that for any $\Psi(t) \in P C\left([-\tau, 0], R^{d}\right)$ with $\Psi\left(0^{-}\right)=\Psi(0)$ the following conditions hold:

(i) $C_{1}\|x\| \leq V(t, x) \leq C_{2}\|x\|$ for all $t \in R_{+}, x \in R^{d}$;

(ii) $D^{+} V(t, \Psi(0)) \leq-m(t) V(t, \Psi(0))$ for all $t \in\left[t_{k}, t_{k+1}\right)$, whenever $V(t, \Psi(0)) \geq V(t+s, \Psi(s)) e^{-\int_{t-\tau}^{t} m(s) d s}$ for $s \in$ $[-\tau, 0]$;

(iii) $V\left(t_{k}, \Psi(0)+I_{k}\left(t_{k}, \Psi(0)\right)\right) \leq\left(1+d_{k}\right) V\left(t_{k}^{-}, \Psi(0)\right)$ for $k=1,2, \ldots$;

(iv) $t_{k}-t_{k-1} \geq l_{1}$ for $k=1,2, \ldots$, and $-\lambda l_{1}+\ln H_{1}<0$, where $H_{1}=\sup _{k}\left\{1+d_{k}\right\}$.

Then the zero solution of (1) is exponentially stable.

Proof. Similar to the proof of Theorem 3.1 in [15], we obtain that

$$
\begin{array}{r}
V(t, x) \leq C_{2} \prod_{i=1}^{k}\left(1+d_{i}\right)\|\Phi\|_{\tau} e^{-\int_{t_{0}}^{t} m(s) d s}, \\
t \in\left[t_{k}, t_{k+1}\right), k=1,2, \ldots .
\end{array}
$$

Since $-\lambda l_{1}+\ln H_{1}<0$, there exists $\alpha$ such that $0<\alpha<\lambda$ and $-(\lambda-\alpha) l_{1}+\ln H_{1}<0$. So

$$
\begin{aligned}
C_{1}\|x\| & \leq V(t, x) \leq C_{2} H_{1}^{k}\|\Phi\|_{\tau} e^{-\lambda\left(t-t_{0}\right)} \\
& \leq C_{2}\|\Phi\|_{\tau} e^{-\lambda\left(t-t_{0}\right)+k \ln H_{1}} \\
& <C_{2}\|\Phi\|_{\tau} e^{-\lambda\left(t-t_{0}\right)+(\lambda-\alpha) k l_{1}} \leq C_{2}\|\Phi\|_{\tau} e^{-\alpha\left(t-t_{0}\right)} .
\end{aligned}
$$

Hence the zero solution of (1) is exponentially stable.
Remark 5. Theorem 3.1 in [15] requires that $d_{i} \geq 0$, and $\sum_{i=0}^{\infty} d_{i}<\infty$, which implies $\lim _{k \rightarrow \infty} d_{k}=0$. In our Theorem 4 , we require $t_{k}-t_{k-1} \geq l_{1}$ for $k=1,2, \ldots$, and $\sup _{k}\left\{1+d_{k}\right\}<e^{\lambda l_{1}}$ instead. This means that the impulsive effects are bounded instead of tending to zero (see Example 13).

Theorem 6. Assume that there exist a function $V \in v_{0}$; constants $d_{k} \in(-1,0), k=1,2, \ldots$; positive constants $l_{1}, l_{2}$, $C_{1}, C_{2}, \lambda$; and a function $m(t) \in \mathrm{PC}\left(\left[t_{0}-\tau, \infty\right), R_{+}\right)$with $\sup _{t \geq t_{0}-\tau} m(t) \leq \lambda$, such that for any $\Psi(t) \in \operatorname{PC}\left([-\tau, 0], R^{d}\right)$ with $\Psi\left(0^{-}\right)=\Psi(0)$, the following conditions hold:

(i) $C_{1}\|x\| \leq V(t, x) \leq C_{2}\|x\|$ for all $t \in R_{+}, x \in R^{d}$;

(ii) $D^{+} V(t, \Psi(0)) \leq m(t) V(t, \Psi(0))$ for all $t \in\left[t_{k}, t_{k+1}\right)$, $k=1,2, \ldots$, whenever $V(t, \Psi(0)) \geq \gamma V(t+s, \Psi(s))$ for $s \in[-\tau, 0]$, where $\gamma$ is a constant and $0<\gamma<$ $H_{2}^{q}, H_{2}=\inf _{k}\left\{1+d_{k}\right\}$ and $q$ is the smallest integer larger than or equal to $\tau / l_{1}$;

(iii) $V\left(t_{k}, \Psi(0)+I_{k}\left(t_{k}, \Psi\right)\right) \leq\left(1+d_{k}\right) V\left(t_{k}^{-}, \Psi(0)\right), k=$ $1,2, \ldots$;

(iv) $l_{1} \leq t_{k}-t_{k-1} \leq l_{2}$ for $k=1,2, \ldots$, and $0<H_{2} \leq H_{1}<$ $e^{-\lambda l_{2}}$, where $H_{1}=\sup _{k}\left\{1+d_{k}\right\}, H_{2}=\inf _{k}\left\{1+d_{k}\right\}$.

Then the zero solution of (1) is exponentially stable.

Proof. Let $x(t)=x\left(t, t_{0}, \Phi\right)$ be the solution of system (1) and $V(t)=V(t, x(t))$. We will prove

$$
\begin{array}{r}
V(t) \leq C_{2} \prod_{i=1}^{k}\left(1+d_{i}\right)\|\Phi\|_{\tau} e^{\int_{t_{0}}^{t} m(s) d s}, \\
t \in\left[t_{k}, t_{k+1}\right), k=1,2, \ldots
\end{array}
$$

Let

$$
Q(t)=\left\{\begin{array}{l}
V(t)-C_{2} \prod_{i=1}^{k}\left(1+d_{i}\right)\|\Phi\|_{\tau} e^{\int_{t_{0}}^{t} m(s) d s}, \\
\quad t \in\left[t_{k}, t_{k+1}\right), \quad k=1,2, \ldots, \\
V(t)-C_{2}\|\Phi\|_{\tau} e^{\int_{t_{0}}^{t} m(s) d s}, \quad t \in\left[t_{0}, t_{1}\right) \\
V(t)-C_{2}\|\Phi\|_{\tau}, \quad t \in\left[t_{0}-\tau, t_{0}\right]
\end{array}\right.
$$

We need to show that $Q(t) \leq 0$ for all $t \geq t_{0}$. It is clear that $Q(t) \leq 0$ for $t \in\left[t_{0}-\tau, t_{0}\right]$, since $Q(t)=V(t)-C_{2}\|\Phi\|_{\tau} \leq 0$ by condition (i).

Next we shall show $Q(t) \leq 0$, for $t \in\left[t_{0}, t_{1}\right)$. Suppose this is not true. Then there is a $t^{*}$ such that $t^{*} \leq \inf \{t \in$ $\left.\left[t_{0}, t_{1}\right), Q(t)>0\right\}, Q\left(t^{*}\right) \leq 0, Q\left(t^{*}\right) \geq(\gamma-1) C_{2}\|\Phi\|_{\tau}$, and

$$
D^{+} Q\left(t^{*}\right)>0 \text {. }
$$


Note that $V\left(t^{*}\right)=Q\left(t^{*}\right)+C_{2}\|\Phi\|_{\tau} e^{\int_{t_{0}}^{t^{*}} m(\sigma) d \sigma}$. Then $V\left(t^{*}+\right.$ $s) \leq C_{2}\|\Phi\|_{\tau} e^{\int_{t_{0}}^{t^{*}} m(\sigma) d \sigma} \leq \gamma^{-1}\left(Q\left(t^{*}\right)+C_{2}\|\Phi\|_{\tau} e^{\int_{t_{0}}^{t^{*}} m(\sigma) d \sigma}\right)=$ $\gamma^{-1} V\left(t^{*}\right)$ for $s \in[-\tau, 0]$. By condition (ii), $D^{+} V\left(t^{*}\right) \leq$ $m\left(t^{*}\right) V\left(t^{*}\right)$. So

$$
\begin{aligned}
D^{+} Q\left(t^{*}\right) & =D^{+} V\left(t^{*}\right)-m\left(t^{*}\right) C_{2}\|\Phi\|_{\tau} e^{\int_{t_{0}}^{t^{*}} m(s) d s} \\
& \leq m\left(t^{*}\right)\left(V\left(t^{*}\right)-C_{2}\|\Phi\|_{\tau} e^{\int_{t_{0}}^{t_{0}^{*}} m(s) d s}\right) \\
& =m\left(t^{*}\right) Q\left(t^{*}\right) \\
& =0,
\end{aligned}
$$

which contradicts (8). Hence $Q(t) \leq 0$, for all $t \in\left[t_{0}, t_{1}\right)$.

Assume that $Q(t) \leq 0$, for $t \in\left[t_{0}, t_{m}\right), m \geq 1$. We shall show that $Q(t) \leq 0$, for $t \in\left[t_{0}, t_{m+1}\right)$. Obviously, by condition (iii)

$$
\begin{aligned}
Q\left(t_{m}\right) & =V\left(t_{m}\right)-C_{2} \prod_{i=1}^{m}\left(1+d_{i}\right)\|\Phi\|_{\tau} e^{\int_{t_{0}}^{t_{m}} m(s) d s} \\
& \leq\left(1+d_{m}\right) V\left(t_{m}^{-}\right)-C_{2} \prod_{i=1}^{m}\left(1+d_{i}\right)\|\Phi\|_{\tau} e^{\int_{t_{0}}^{t_{m}} m(s) d s} \\
& =\left(1+d_{m}\right) Q\left(t_{m}^{-}\right) \\
& \leq 0
\end{aligned}
$$

Suppose that there exists a $t$ such that $t \in\left[t_{m}, t_{m+1}\right)$ and $Q(t)>0$. There is a $t^{*}$ such that $t^{*} \leq \inf \left\{t \in\left[t_{m}, t_{m}+\right.\right.$ 1), $Q(t)>0\}, Q\left(t^{*}\right) \leq 0, Q\left(t^{*}\right) \geq\left(\gamma H_{2}^{-q}-1\right) C_{2} \prod_{i=1}^{m}(1+$ $\left.d_{i}\right)\|\Phi\|_{\tau}$, and

$$
D^{+} Q\left(t^{*}\right)>0 \text {. }
$$

Since $V\left(t^{*}\right)=Q\left(t^{*}\right)+C_{2} \prod_{i=1}^{m}\left(1+d_{i}\right)\|\Phi\|_{\tau} e^{\int_{t_{0}}^{t^{*}} m(s) d s}$, then for any $s \in[-\tau, 0]$, we have

$$
\begin{aligned}
V\left(t^{*}+s\right) & \leq Q\left(t^{*}+s\right)+C_{2} \prod_{i=1}^{m-q}\left(1+d_{i}\right)\|\Phi\|_{\tau} e^{\int_{t_{0}}^{t^{*}+s} m(\sigma) d \sigma} \\
& \leq H_{2}^{-q} C_{2} \prod_{i=1}^{m}\left(1+d_{i}\right)\|\Phi\|_{\tau} e^{\int_{t_{0}}^{t^{*}} m(\sigma) d \sigma} \\
& \leq \gamma^{-1}\left(Q\left(t^{*}\right)+C_{2} \prod_{i=1}^{m}\left(1+d_{i}\right)\|\Phi\|_{\tau} e^{\int_{t_{0}}^{t^{*}} m(\sigma) d \sigma}\right) \\
& \leq \gamma^{-1} V\left(t^{*}\right) .
\end{aligned}
$$

Thus by condition (ii), $D^{+} V\left(t^{*}\right) \leq m\left(t^{*}\right) V\left(t^{*}\right)$, then

$$
\begin{aligned}
D^{+} Q\left(t^{*}\right) & =D^{+} V\left(t^{*}\right)+m\left(t^{*}\right) C_{2} \prod_{i=1}^{m}\left(1+d_{i}\right)\|\Phi\|_{\tau} e^{\int_{t_{0}}^{t^{*}} m(s) d s} \\
& \leq m\left(t^{*}\right)\left(V\left(t^{*}\right)-C_{2} \prod_{i=1}^{m}\left(1+d_{i}\right)\|\Phi\|_{\tau} e^{\int_{t_{0}}^{t^{*}} m(s) d s}\right) \\
& =m\left(t^{*}\right) Q\left(t^{*}\right) \\
& =0,
\end{aligned}
$$

which contradicts (11). Hence $Q(t) \leq 0$ for all $t \in\left[t_{m}, t_{m+1}\right)$. By induction, $Q(t) \leq 0$ for all $t \geq t_{0}$. In view of $m(t) \leq \lambda$ for all $t \geq t_{0}-\tau$, we obtain

$$
\begin{aligned}
V(t) & \leq C_{2} \prod_{i=1}^{k}\left(1+d_{i}\right)\|\Phi\|_{\tau} e^{\int_{t_{0}}^{t} m(s) d s} \\
& \leq C_{2}\|\Phi\|_{\tau} H_{1}^{k} e^{\int_{t_{0}}^{t} m(s) d s} \\
& \leq C_{2} H_{1}^{k}\|\Phi\|_{\tau} e^{\lambda\left(t-t_{0}\right)},
\end{aligned}
$$

for $t \in\left[t_{k}, t_{k+1}\right), k=1,2, \ldots$ Since $H_{1}<e^{-\lambda l_{2}}$, there exists $\alpha$ such that $0<\alpha<\lambda$ and $\lambda l_{2}+\ln H_{2}<-\alpha l_{2}$. By condition (i)

$$
\begin{aligned}
C_{1}\|x\| & \leq V(t, x) \\
& \leq C_{2} H_{1}^{k}\|\Phi\|_{\tau} e^{\lambda\left(t-t_{0}\right)} \leq C_{2}\|\Phi\|_{\tau} e^{\lambda\left(t-t_{0}\right)+k \ln H_{1}} \\
& \leq C_{2}\|\Phi\|_{\tau} e^{\lambda\left(t-t_{0}\right)-(\lambda+\alpha) k l_{2}} \leq M\|\Phi\|_{\tau} e^{-\alpha\left(t-t_{0}\right)},
\end{aligned}
$$

where $M=C_{2} e^{(\lambda+\alpha) l_{2}}$. Hence the zero solution of (1) is exponentially stable.

Remark 7. Theorem 6 says that the delay differential equation is unstable and the suitable impulse effects are given, then it will become stable (see Example 14). Compared with the Theorem 3.1 in [16], we do not require that $\tau \leq t_{k}-t_{k-1}$. For example, by Theorem 6 we know that the zero solution of the following system is exponentially stable:

$$
\begin{aligned}
x^{\prime}(t)= & \frac{1}{2} x(t)+\frac{1}{8} x(t-1), \\
& t \geq 0, t \neq t_{k}, t_{k}=\frac{k}{2}, k=1,2, \ldots, \\
x\left(t_{k}\right)= & \frac{1}{3} x\left(t_{k}^{-}\right), \quad t_{k}=\frac{k}{2}, k=1,2, \ldots
\end{aligned}
$$

In the following we consider

$$
\begin{aligned}
x^{\prime}(t)= & a x(t)+b x(t-\tau), \\
& t \geq 0, t \in[(k-1) \tau, k \tau) k=1,2, \ldots, \\
x\left(t_{k}\right)= & \left(1+c_{k}\right) x\left(t_{k}^{-}\right), \quad t_{k}=k \tau, k=1,2, \ldots,
\end{aligned}
$$

where $\tau>0$, and $d_{k}, a, b \in R$ are constants. 
Theorem 8. Assume that $c_{k} \neq-1, k=1,2, \ldots$, and there is a constant $\lambda>0$ such that $a+|b| e^{\lambda \tau} \leq-\lambda$ and $0<H_{1}<e^{\lambda \tau}$, where $H_{1}=\sup _{k}\left\{\left|1+c_{k}\right|\right\}$. Then the zero solution of (17) is exponentially stable.

Proof. Assume that $V(x)=V(t, x)=|x|$.

(i) Obviously, there exist $C_{1}=C_{2}=1$, such that $C_{1}|x| \leq$ $V(x) \leq C_{2}|x|$.

(ii) Assume that $m(t)=\lambda$ for all $t \geq-\tau$. For any $\Psi \in$ $\mathrm{PC}([-\tau, 0], R)$, if

$$
\begin{aligned}
V(t, \Psi(0)) & \geq V(t+s, \Psi(s)) e^{-\int_{t-\tau}^{t} m(s) d s} \\
& =V(t+s, \Psi(s)) e^{-\lambda \tau}
\end{aligned}
$$

we have $|\Psi(-\tau)| \leq e^{\lambda \tau}|\Psi(0)|$. For $s \in[-\tau, 0]$, we have

$$
\begin{aligned}
D^{+} V(t, \Psi(0)) & \leq a|\Psi(0)|+|b||\Psi(-\tau)| \\
& \leq a|\Psi(0)|+|b| e^{\lambda \tau}|\Psi(0)| \\
& =\left(a+|b| e^{\lambda \tau}\right)|\Psi(0)| \\
& \leq-\lambda|\Psi(0)| \\
& =-m(t) V(t, \Psi(0)) .
\end{aligned}
$$

(iii) Suppose that $1+d_{k}=\left|\left(1+c_{k}\right)\right|$. Hence

$$
\begin{aligned}
V\left(x\left(t_{k}\right)\right) & =\left|x\left(t_{k}\right)\right|=\left|\left(1+c_{k}\right)\right|\left|x\left(t_{k}^{-}\right)\right| \\
& =\left(1+d_{k}\right)\left|x\left(t_{k}^{-}\right)\right|=\left(1+d_{k}\right) V\left(x\left(t_{k}^{-}\right)\right) .
\end{aligned}
$$

(iv) Obviously, $l_{1}=\tau=t_{k}-t_{k-1}$ and $-\lambda l_{1}+\ln H_{1}<0$.

By Theorem 4, the zero solution of (11) is exponentially stable.

Similarly, by Theorem 6 we have the following theorem.

Theorem 9. Assume that $0<\left|1+c_{k}\right|<1, k=1,2, \ldots$, and there are constants $\lambda$ and $\gamma$ such that $\lambda>0,0<\gamma<H_{2} \leq H_{1}<$ $e^{-\lambda \tau}$, and $a+|b| \gamma^{-1} \leq \lambda$, where $H_{1}=\sup _{k}\left\{\left|1+c_{k}\right|\right\}, H_{2}=$ $\inf _{k}\left\{\left|1+c_{k}\right|\right\}$. Then the zero solution of (17) is exponentially stable.

\section{The Euler Method for Linear IDDEs}

In this section, we consider the exponential stability of the Euler method for (17). The convergence property can be proved similarly to [4]. The Euler method for (17) with initial function $\Phi \in \operatorname{PC}([-\tau, 0], R)$ is given by

$$
\begin{gathered}
x_{k, l+1}=x_{k, l}+\operatorname{hax}_{k, l}+h b x_{k-1, l}, \\
l=0,1, \ldots, m-1, k=0,1, \ldots, \\
x_{(k+1), 0}=\left(1+c_{k+1}\right) x_{k, m}, \quad k=0,1,2, \ldots, \\
x_{-1, l}=\Phi(-\tau+l h),
\end{gathered}
$$

where $h=\tau / m$. Let $n=k m+l$, then $x_{n}=x_{k m+l}=x_{k, l}$ is an approximation for the exact solution $x((k m+l) h)$ for $k=$ $0,1,2, \ldots, l=0,1,2, \ldots, m-1$, and $x_{k, m}$ is an approximation for $x\left((k+1) \tau^{-}\right)$.

Definition 10. The Euler method for (11) is said to be exponentially stable if there exist positive constants $\lambda, M$, and $M_{1}$, for any $\Phi \in \operatorname{PC}([-\tau, 0], R)$, such that $\left\|x_{n}\right\| \leq M\|\Phi\|_{\tau} e^{-n \lambda h}$ for $h=\tau / m, m \geq M_{1}$, and $n=1,2, \ldots$.

The following theorem indicates that the Euler method preserves the property of exact solutions which was obtained above.

Theorem 11. Under the conditions of Theorem 8, the Euler method for (17) is exponentially stable.

Proof. If $a<0$, then $M_{1}=-a \tau$. (i) If $H_{1}>1$, we want to prove that

$$
\left|x_{k, l}\right| \leq\|\Phi\|_{\tau} H_{1}^{k} e^{-(k m+l) \lambda h}
$$

for $k=0,1,2, \ldots, l=0,1,2, \ldots, m$. Obviously, $\left|x_{0,0}\right|=$ $|\Phi(0)| \leq\|\Phi\|_{\tau}$. Firstly, we consider the case $k=0$ and $l=1$. Because $h=\tau / m$ and $m \geq M_{1}$, so $1+h a \geq 0$. Hence we have

$$
\begin{aligned}
\left|x_{0,1}\right| & =\left|x_{0,0}+h a x_{0,0}+h b x_{-1,0}\right| \\
& \leq(1+h a)\left|x_{0,0}\right|+h|b|\left|x_{-1,0}\right| \\
& \leq(1+h a)\|\Phi\|_{\tau}+h|b|\|\Phi\|_{\tau} \\
& \leq\left(1+h a+h|b| e^{\lambda \tau}\right)\|\Phi\|_{\tau} .
\end{aligned}
$$

Because $a+|b| e^{\lambda \tau} \leq-\lambda$, we have $\left|x_{0,1}\right| \leq(1-h \lambda)\|\Phi\|_{\tau}$. By the inequality $e^{-x} \geq 1-x$ holding for all $x \in R$, we get $\left|x_{0,1}\right| \leq$ $\|\Phi\|_{\tau} e^{-\lambda h}$.

Assume that $\left|x_{0, p}\right| \leq\|\Phi\|_{\tau} e^{-\lambda p h}$ for $p<l \leq m$. Then

$$
\begin{aligned}
\left|x_{0, l}\right| & =\left|x_{0, l-1}+h a x_{0, l-1}+h b x_{-1, l-1}\right| \\
& \leq(1+h a)\left|x_{0, l-1}\right|+h|b|\left|x_{-1, l-1}\right| \\
& \leq(1+h a)\|\Phi\|_{\tau} e^{-\lambda(l-1) h}+h|b|\|\Phi\|_{\tau} \\
& \leq\left(1+h a+h|b| e^{\lambda \tau}\right)\|\Phi\|_{\tau} e^{-\lambda(l-1) h} \\
& \leq(1-h \lambda)\|\Phi\|_{\tau} e^{-\lambda(l-1) h} \\
& \leq\|\Phi\|_{\tau} e^{-\lambda l h} .
\end{aligned}
$$

So (22) holds for $k=0, l=0,1,2, \ldots, m$. Suppose that (22) holds for $n<k, l=0,1,2, \ldots, m$. Next, we shall prove (22) holds, when $n=k, l=0,1,2, \ldots, m$. Hence

$$
\left|x_{k, 0}\right|=\left|1+c_{k}\right|\left|x_{k-1, m}\right| \leq\|\Phi\|_{\tau} H_{1}^{k} e^{-k m \lambda h} .
$$




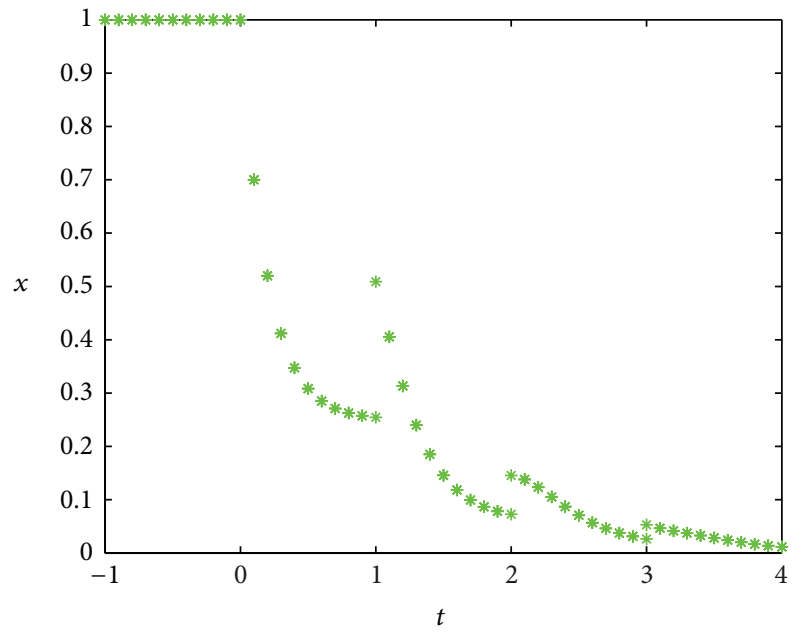

FIGURE 1: The solutions of (29), as $\Phi \equiv 1, h=1 / 10$.

Assume that $\left|x_{k, u}\right| \leq\|\Phi\|_{\tau} H_{1}^{k} e^{-(k m+u) \lambda h}$ for $u<l \leq m$. Then

$$
\begin{aligned}
\left|x_{k, l}\right|= & \left|x_{k, l-1}+h a x_{k, l-1}+h b x_{k-1, l-1}\right| \\
\leq & (1+h a)\left|x_{k, l-1}\right|+h|b|\left|x_{k-1, l-1}\right| \\
\leq & (1+h a)\|\Phi\|_{\tau} H_{1}^{k} e^{-(k m+l-1) \lambda h} \\
& +h|b| \cdot\|\Phi\|_{\tau} H_{1}^{k-1}\left|1+d_{i}\right| e^{-(k m-m+l-1) \lambda h} \\
\leq & \left(1+h a+h|b| e^{\lambda \tau}\right)\|\Phi\|_{\tau} H_{1}^{k} e^{-(k m+l-1) \lambda h} \\
\leq & (1-h \lambda)\|\Phi\|_{\tau} H_{1}^{k} e^{-(k m+l-1) \lambda h} \\
\leq & \|\Phi\|_{\tau} H_{1}^{k} e^{-(k m+l) \lambda h} .
\end{aligned}
$$

Hence (22) holds. Since $-\lambda l_{1}+\ln H_{1}<0$, there exists $\alpha$ such that $0<\alpha<\lambda$ and $-(\lambda-\alpha) l_{1}+\ln H_{1}<0$. Hence

$$
\begin{aligned}
\left|x_{k, l}\right| & \leq\|\Phi\|_{\tau} H_{1}^{k} e^{-(k m+l) \lambda h} \leq\|\Phi\|_{\tau}\left(e^{\ln H_{1}-\lambda m h}\right)^{k} e^{-\lambda l h} \\
& \leq\|\Phi\|_{\tau} e^{-\alpha(m k+l) h} .
\end{aligned}
$$

(ii) If $H_{1} \leq 1$, we can prove that

$$
\left|x_{k, l}\right| \leq\|\Phi\|_{\tau} e^{-\lambda(k m+l) h}
$$

Consequently, the theorem holds.

Theorem 12. Under the conditions of Theorem 9, the Euler method for (17) is exponentially stable.

Example 13. Consider the system

$$
\begin{aligned}
& x^{\prime}(t)=-4 x(t)+x(t-1), \quad t \geq 0, t \neq k, k=1,2, \ldots, \\
& x(k)=2 x\left(k^{-}\right), \quad k=1,2, \ldots
\end{aligned}
$$

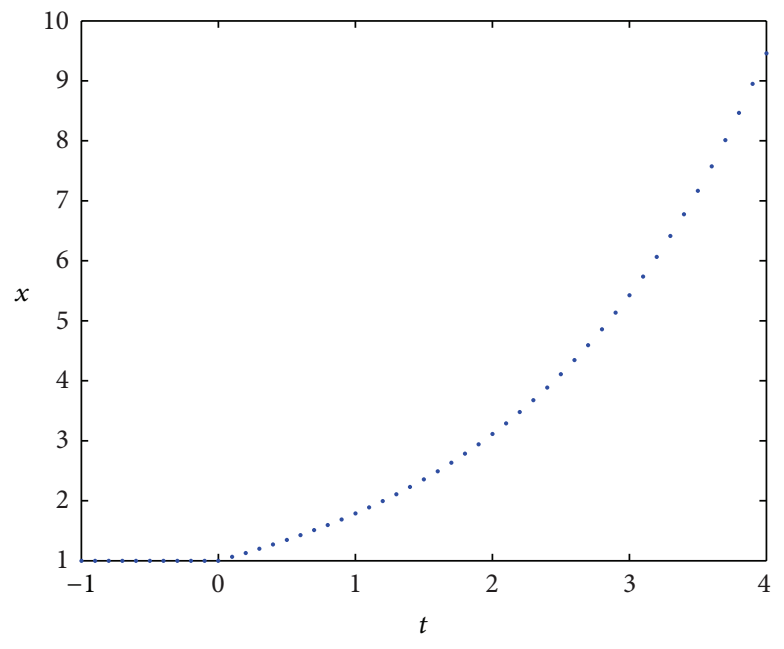

FIGURE 2: The solution of $(30)$ as $\Phi(t) \equiv 1, t \in[-\tau, 0]$.

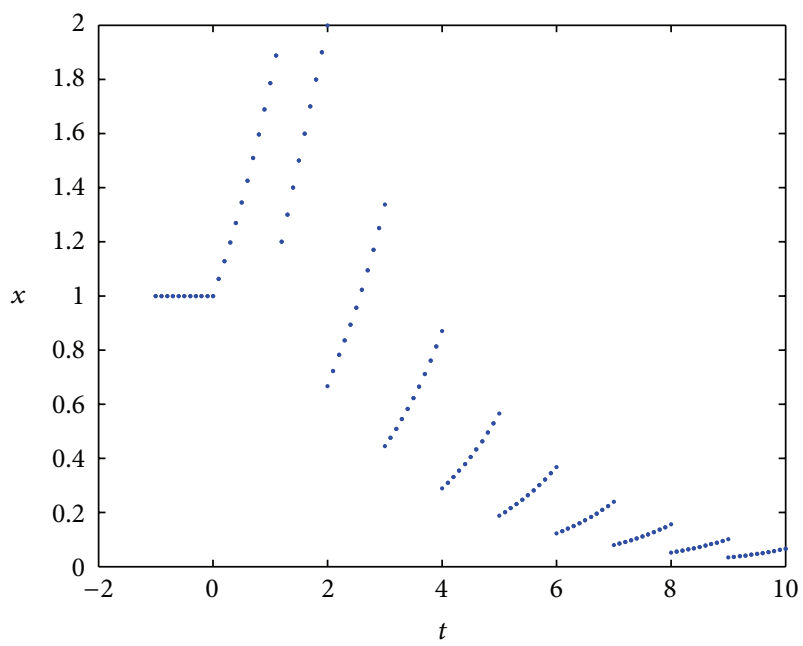

FIGURE 3: The solutions of (31), as $\Phi \equiv 1, h=1 / 10$.

Obviously, $\lambda=1$ satisfies the Theorem 8 . Therefore the zero solution of (29) is exponentially stable. By Theorem 11, the Euler method for (29) is also exponentially stable (see Figure 1).

Example 14. Obviously, the zero solution of the system

$$
x^{\prime}(t)=\frac{1}{2} x(t)+\frac{1}{8} x(t-1), \quad t \geq 0,
$$

is unstable (see Figure 2) while the zero solution of the following system

$$
\begin{gathered}
x^{\prime}(t)=\frac{1}{2} x(t)+\frac{1}{8} x(t-1), \quad t \geq 0, t \neq k, k=1,2, \ldots, \\
x(k)=\frac{1}{3} x\left(k^{-}\right), \quad k=1,2, \ldots,
\end{gathered}
$$


is exponentially stable by Theorem 9 with $\lambda=1$. By Theorem 12, the Euler method for (31) is also exponentially stable (see Figure 3).

\section{Acknowledgments}

The authors wish to thank referees for valuable comments. The research was supported by the NSF of China no. 11071050 .

\section{References}

[1] A. Anokhin, L. Berezansky, and E. Braverman, "Exponential stability of linear delay impulsive differential equations," Journal of Mathematical Analysis and Applications, vol. 193, no. 3, pp. 923-941, 1995.

[2] G. Ballinger and X. Liu, "Existence and uniqueness results for impulsive delay differential equations," Dynamics of Continuous, Discrete and Impulsive Systems, vol. 5, no. 1-4, pp. 579-591, 1999.

[3] L. Berezansky and L. Idels, "Exponential stability of some scalar impulsive delay differential equations," Communications in Applied Analysis, vol. 2, no. 3, pp. 301-308, 1998.

[4] X. Ding, K. Wu, and M. Liu, "The Euler scheme and its convergence for impulsive delay differential equations," Applied Mathematics and Computation, vol. 216, no. 5, pp. 1566-1570, 2010.

[5] M. De La Sen and N. Luo, "A note on the stability of linear time-delay systems with impulsive inputs," IEEE Transactions on Circuits and Systems. I, vol. 50, no. 1, pp. 149-152, 2003.

[6] M. De la Sen, "Stability of impulsive time-varying systems and compactness of the operators mapping the input space into the state and output spaces," Journal of Mathematical Analysis and Applications, vol. 321, no. 2, pp. 621-650, 2006.

[7] X. Liu, "Stability results for impulsive differential systems with applications to population growth models," Dynamics and Stability of Systems, vol. 9, no. 2, pp. 163-174, 1994.

[8] X. Liu, X. Shen, and Y. Zhang, "Exponential stability of singularly perturbed systems with time delay," Applicable Analysis, vol. 82, no. 2, pp. 117-130, 2003.

[9] X. Liu and Q. Wang, "The method of Lyapunov functionals and exponential stability of impulsive systems with time delay," Nonlinear Analysis: Theory, Methods \& Applications, vol. 66, no. 7, pp. 1465-1484, 2007.

[10] X. Liu, X. Shen, Y. Zhang, and Q. Wang, "Stability criteria for impulsive systems with time delay and unstable system matrices," IEEE Transactions on Circuits and Systems. I, vol. 54, no. 10, pp. 2288-2298, 2007.

[11] Z. Luo and J. Shen, "Stability of impulsive functional differential equations via the Liapunov functional," Applied Mathematics Letters, vol. 22, no. 2, pp. 163-169, 2009.

[12] I. M. Stamova and G. T. Stamov, "Lyapunov-Razumikhin method for impulsive functional differential equations and applications to the population dynamics," Journal of Computational and Applied Mathematics, vol. 130, no. 1-2, pp. 163-171, 2001.

[13] J. H. Shen, "Razumikhin techniques in impulsive functionaldifferential equations," Nonlinear Analysis: Theory, Methods \& Applications, vol. 36, no. 1, pp. 119-130, 1999.
[14] J. Shen and J. Yan, "Razumikhin type stability theorems for impulsive functional-differential equations," Nonlinear Analysis: Theory, Methods \& Applications, vol. 33, no. 5, pp. 519-531, 1998.

[15] Q. Wang and X. Liu, "Exponential stability for impulsive delay differential equations by Razumikhin method," Journal of Mathematical Analysis and Applications, vol. 309, no. 2, pp. 462473, 2005.

[16] Q. Wang and X. Liu, "Impulsive stabilization of delay differential systems via the Lyapunov-Razumikhin method," Applied Mathematics Letters, vol. 20, no. 8, pp. 839-845, 2007.

[17] Y. Zhang and J. Sun, "Stability of impulsive functional differential equations," Nonlinear Analysis: Theory, Methods \& Applications, vol. 68, no. 12, pp. 3665-3678, 2008.

[18] G. L. Zhang, M. H. Song, and M. Z. Liu, "Asymptotical stability of a class impulsive delay differential equations," Journal of Applied Mathematics, vol. 2012, Article ID 723893, 9 pages, 2012.

[19] V. Covachev, H. Akça, and F. Yeniçerioğlu, "Difference approximations for impulsive differential equations," Applied Mathematics and Computation, vol. 121, no. 2-3, pp. 383-390, 2001.

[20] M. Z. Liu, H. Liang, and Z. W. Yang, "Stability of RungeKutta methods in the numerical solution of linear impulsive differential equations," Applied Mathematics and Computation, vol. 192, no. 2, pp. 346-357, 2007.

[21] X. J. Ran, M. Z. Liu, and Q. Y. Zhu, "Numerical methods for impulsive differential equation," Mathematical and Computer Modelling, vol. 48, no. 1-2, pp. 46-55, 2008. 


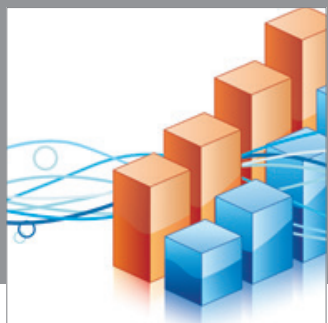

Advances in

Operations Research

mansans

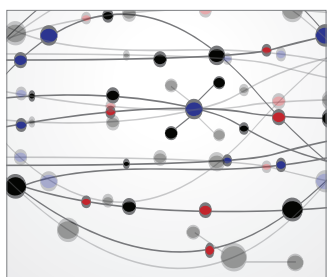

The Scientific World Journal
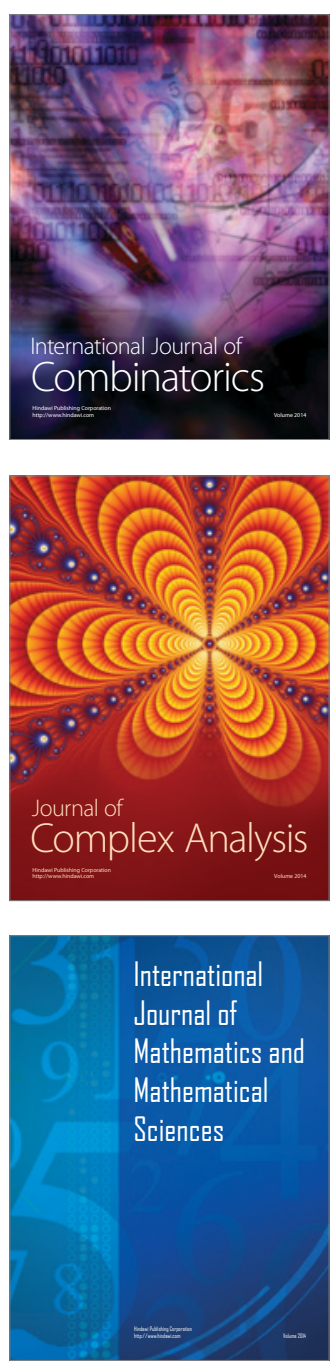
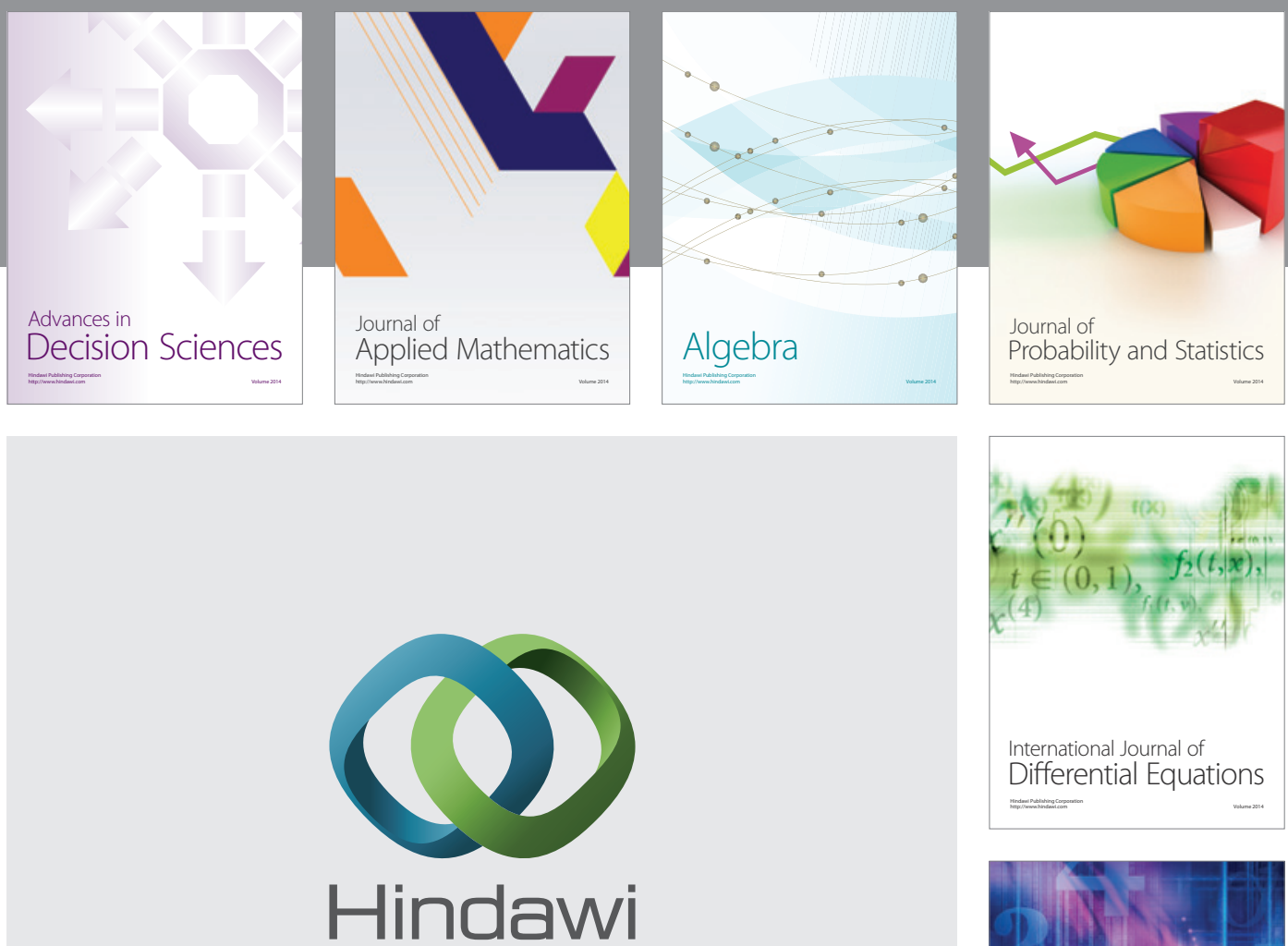

Submit your manuscripts at http://www.hindawi.com
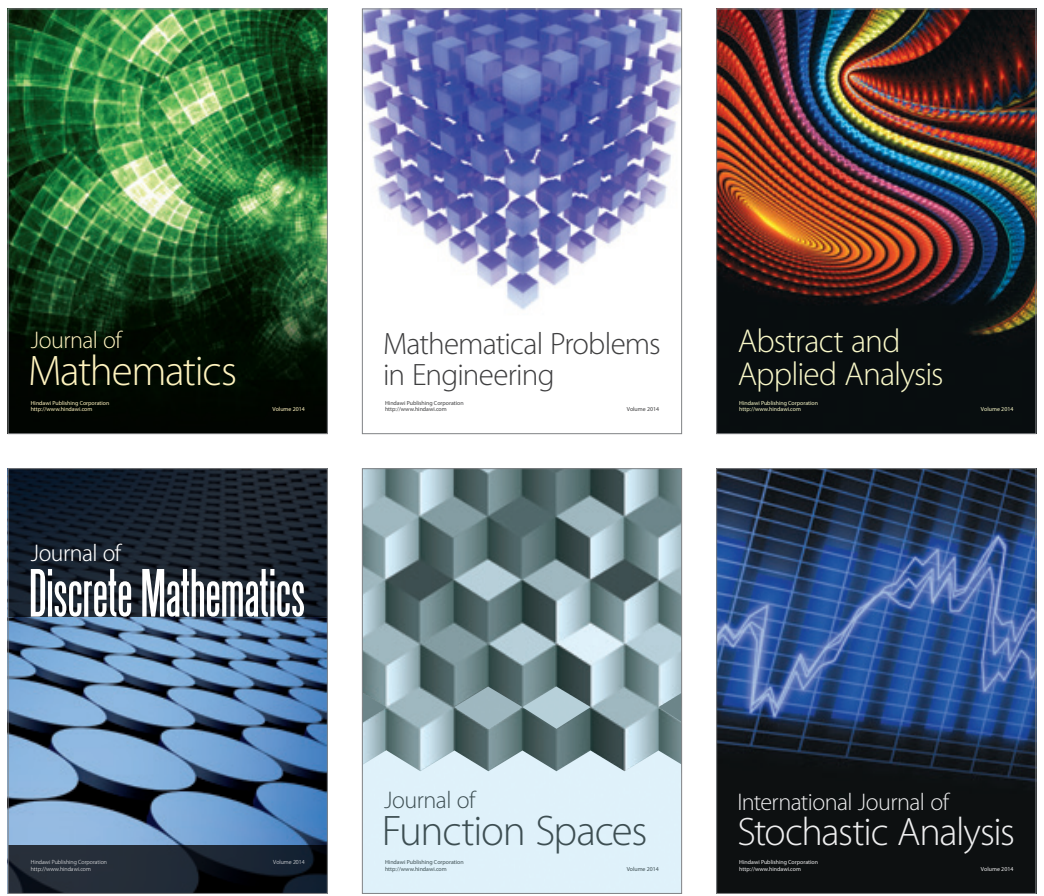

Journal of

Function Spaces

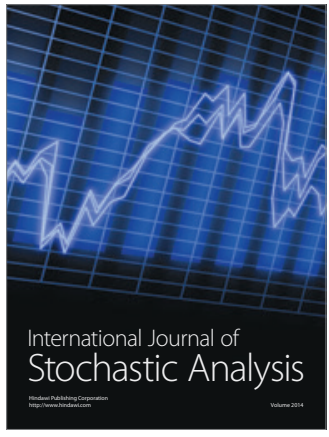

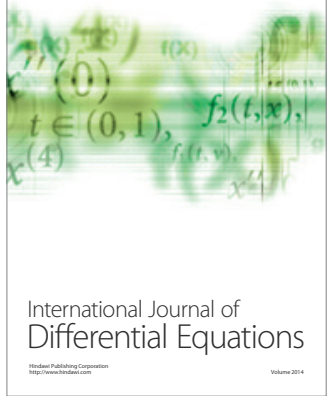
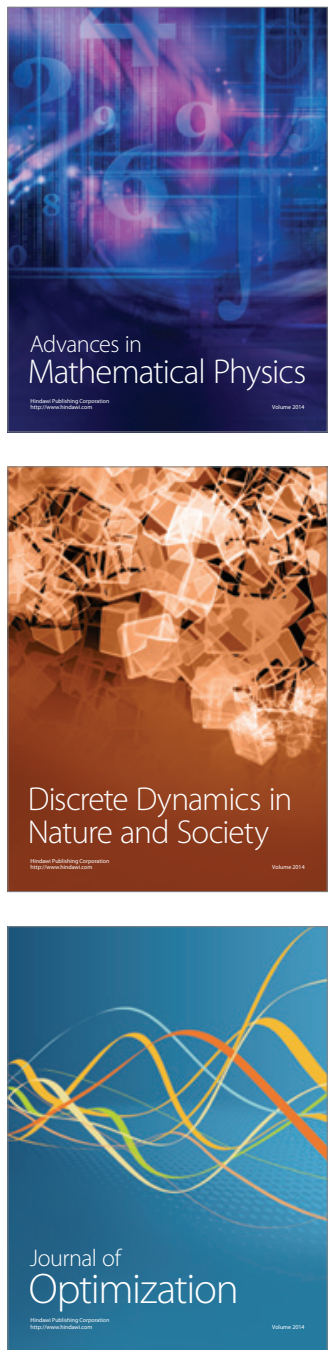